\title{
One hundred consecutive endoscopic repairs of sagittal craniosynostosis: an evolution in care
}

\author{
Dennis C. Nguyen, MD, ${ }^{1}$ Scott J. Farber, MD, ${ }^{1}$ Gary B. Skolnick, BS, ${ }^{1}$ \\ Sybill D. Naidoo, PhD, RN, CPNP, ${ }^{1}$ Matthew D. Smyth, MD, ${ }^{2}$ Alex A. Kane, MD, ${ }^{3}$ \\ Kamlesh B. Patel, MD, ${ }^{1}$ and Albert S. Woo, MD ${ }^{4}$ \\ 'Division of Plastic and Reconstructive Surgery, Department of Surgery, and 2Division of Pediatric Neurosurgery, Department \\ of Neurosurgery, Washington University School of Medicine, St. Louis, Missouri; ${ }^{3}$ Department of Plastic Surgery, University of \\ Texas Southwestern Medical Center, Dallas, Texas; and ${ }^{4}$ Division of Plastic and Reconstructive Surgery, The Warren Alpert \\ Medical School of Brown University, Providence, Rhode Island
}

OBJECTIVE Endoscope-assisted repair of sagittal craniosynostosis was adopted at St. Louis Children's Hospital in 2006. This study examines the first 100 cases and reviews the outcomes and evolution of patient care protocols at our institution.

METHODS The authors performed a retrospective chart review of the first 100 consecutive endoscopic repairs of sagittal craniosynostosis between 2006 and 2014. The data associated with length of hospital stay, blood loss, transfusion rates, operative times, cephalic indices (Cls), complications, and cranial remolding orthosis were reviewed. Measurements were taken from available preoperative and 1-year postoperative 3D reconstructed CT scans.

RESULTS The patients' mean age at surgery was $3.3 \pm 1.1$ months. Of the 100 patients, 30 were female and 70 were male. The following perioperative data were noted. The mean operative time $( \pm S D)$ was $77.1 \pm 22.2$ minutes, the mean estimated blood loss was $34.0 \pm 34.8 \mathrm{ml}$, and the mean length of stay was $1.1 \pm 0.4$ days; $9 \%$ of patients required transfusions; and the mean pre- and postoperative $\mathrm{Cl}$ values were $69.1 \pm 3.8$ and $77.7 \pm 4.2$, respectively. Conversion to open technique was required in 1 case due to presence of a large emissary vein that was difficult to control endoscopically. The mean duration of helmet therapy was $8.0 \pm 2.9$ months. Parietal osteotomies were eventually excluded from the procedure.

CONCLUSIONS The clinical outcomes and improvements in $\mathrm{Cl}$ seen in our population are similar to those seen at other high-volume centers. Since the inception of endoscope-assisted repair at our institution, the patient care protocol has undergone several significant changes. We have been able to remove less cranium using our "narrow-vertex" suturectomy technique without affecting patient safety or outcome. Patient compliance with helmet therapy and collaborative care with the orthotists remain the most essential aspects of a successful outcome.

https://thejns.org/doi/abs/10.3171/2017.5.PEDS16674

KEY WORDS craniosynostosis; endoscopic; craniofacial surgery

$\mathrm{S}$ AGITTAL craniosynostosis remains the most common form of premature single-suture fusion. ${ }^{13}$ The primary goals of reconstruction are to relieve growth restriction and improve head shape (e.g., biparietal narrowing, frontooccipital bossing), while also minimizing morbidity. Surgical techniques and care for patients with scaphocephaly have evolved over the last century. Surgical management of this disorder was initially introduced in the 1890 s by Lannelongue ${ }^{17}$ and Lane. ${ }^{16}$ Since then, meth- ods have evolved from the original strip craniectomy to a spectrum of partial or complete cranial vault remodeling. ${ }^{19}$ More complex reconstructive techniques, such as total calvarial vault remodeling and the pi procedure, have produced excellent results, but can involve substantial morbidity. ${ }^{14}$ As an alternative to these more aggressive procedures, endoscope-assisted suturectomy was pioneered in 1998 by Jimenez and Barone ${ }^{9}$ and purportedly provided the benefits of shorter surgery, limited blood loss, lower 
overall cost of care, and decreased hospital stay. However, with this procedure, much of the final result is dependent upon postoperative molding helmet therapy, which is required of all patients to limit anteroposterior projection and optimize parietal expansion. ${ }^{12}$ The endoscopic technique was adopted by our institution in 2006. This study examines the first 100 endoscope-assisted suturectomy and postoperative helmeting cases for sagittal synostosis and describes outcomes and the evolution of patient care protocols at our institution.

\section{Methods}

This study was approved by the institutional review board of the Washington University School of Medicine in St. Louis. A retrospective chart review was performed of the first 100 consecutive endoscopic repairs of sagittal craniosynostosis between 2006 and 2014 at St. Louis Children's Hospital. Hospital records were reviewed and data associated with length of stay, blood loss, transfusion rates, operative times, cephalic indices (CIs), complications, and cranial remolding orthoses were collected. Measurements were taken from available preoperative and 1-year postoperative 3D reconstructed CT scans. All scans were performed using a low-dose radiation protocol. Statistical analyses were performed using SPSS, version 20 (IBM Corp.). Significance was determined by a value of $\mathrm{p}=0.05$.

\section{Technique}

Our institution offers both open and endoscope-assisted repair for all patients with isolated sagittal synostosis who can have surgery scheduled by 5 months of age. Currently, roughly $90 \%$ of parents elect for endoscopic repair during this time. Thereafter, open repair is typically recommended for any patient presenting older than 5 months of age, although the endoscopic technique is occasionally used in infants as old as 6-7 months for select cases of milder deformities. Although not specifically addressed in this paper, since 2003, our patients who underwent open repair received a modified pi cranial vault reconstruction. Initially, we performed this technique combined with bifrontal craniotomy and later transitioned away from bifrontal manipulation. ${ }^{15}$

Endoscope-assisted wide-vertex craniectomies with bilateral parietal wedge osteotomies were initially performed as previously described by Jimenez and Barone and colleagues. ${ }^{9-11}$ The patient is placed prone in a modified sphinx position on either a padded beanbag or DORO Headrest System (Pro Med Instruments) (Fig. 1). A 2- to $4-\mathrm{cm}$ transverse midline incision is made just posterior to the anterior fontanelle. A second incision of the same size and orientation is made just anterior to the lambda. Subgaleal dissection is performed with the aid of an endoscope. Once the strip of calvaria is exposed and outlined, transverse osteotomies are made and a $30^{\circ}$ rigid endoscope is

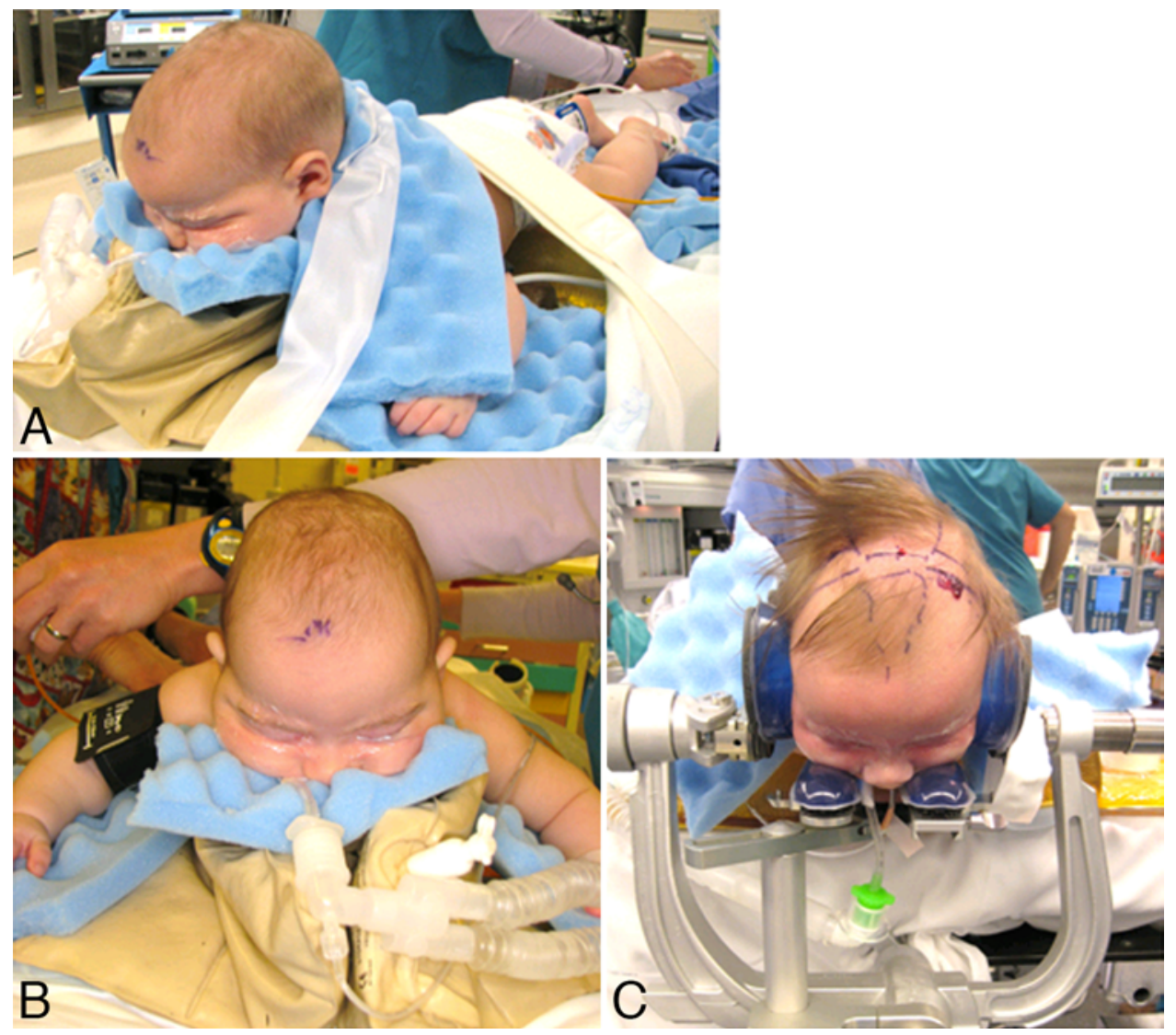

FIG. 1. A and B: Patient positioned prone with foam pads and beanbag. C: Use of DORO head holder to stabilize head and airway. Figure is available in color online only. 
used to guide careful dissection of the dura off the strip to be removed. Once fully dissected free of the dura, the strip containing the fused sagittal suture is removed by making 2 paramedian osteotomies. Due to the softness of the pediatric cranium, Tessier bone-cutting scissors (KLS Martin, LP) are used for the craniectomy. The craniectomy is between 4 and $5 \mathrm{~cm}$ wide and is performed in conjunction with bilateral wedge osteotomies posterior to the coronal sutures and anterior to the lambdoid sutures. Hemostasis is achieved with bipolar cautery of the dura, monopolar coagulation of the bone edges, and application of Floseal hemostatic matrix, and then the small incisions are closed with resorbable sutures (Fig. 2).

Starting in early 2011, patients began receiving a narrow-vertex suturectomy without bilateral wedge osteotomies, a procedure described at Boston Children's Hospital..$^{1,2}$ The incision width is smaller (between 1.5 and 2.5 $\mathrm{cm}$ ), and the osteotomy width is about $2 \mathrm{~cm}$-just enough to incorporate the fused suture (Fig. 2). ${ }^{4}$

\section{Helmet Protocol}

Our helmet protocol has been previously described..$^{20}$ We advocate use of a molding helmet to allow the expanding forces of the growing brain to be guided by the exter-

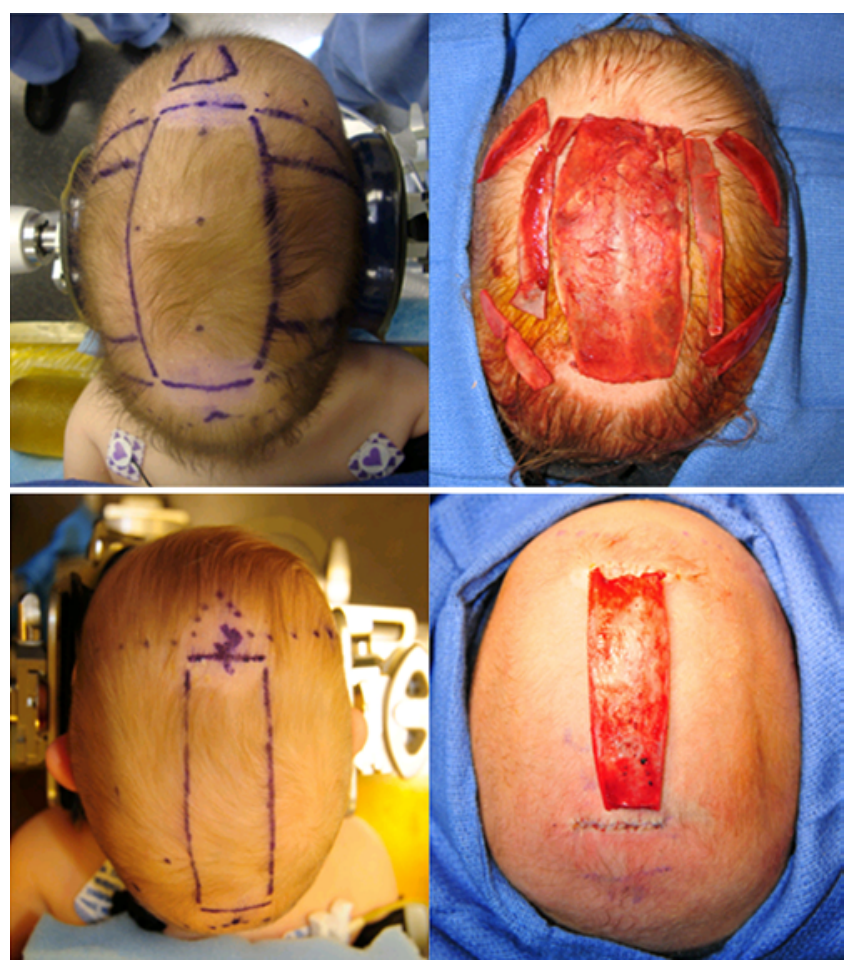

FIG. 2. Comparison of wide-vertex craniectomy and barrel-stave osteotomies (upper) and narrow-vertex craniectomy without barrel-stave osteotomies (lower). The preoperative photographs (left) show the skin markings for the surgical procedures. The postoperative photographs (right) show the removed bone fragments placed on top of the patients' skulls. Two small incisions (3-4 cm in length) are made over the bregma (anterior) and lambda (posterior) for surgical access. The craniotomy and epidural dissection are performed under endoscopic guidance. A segment of bone containing the fused sagittal suture is removed. Figure is available in color online only. nal forces of the helmet. This process realigns calvarial growth from the scaphocephalic appearance to a more normal head shape, by encouraging biparietal expansion. The patient is seen by the orthotist preoperatively when possible, and a custom helmet is prepared such that therapy can be initiated as soon as 1-3 days after the operation. Specific to patients with sagittal craniosynostosis, the helmet is designed to provide direct contact to the frontal and occipital midline regions, while contact to the lateral regions is avoided. Attention should also be given to the vertex to prevent a turricephaly or a "volcano hump" upon completion of helmet therapy. Our aim is to achieve both a CI within the normal range (75-85) and an overall good aesthetic outcome (Fig. 3). The helmet is worn at least 23 hours per day until the patient is 1 year old, when $65 \%$ of the brain's growth has occurred. ${ }^{23}$ During this 1st year following surgery, $2-4$ orthotic helmets are required to account for changes in cranial shape and size.

Communication between the surgical team and the orthotist is important to success, as the result is dependent on the quality of molding helmet therapy. This was especially true in our case series because 2 orthotic companies were used: one to accommodate patients who lived locally and a second national chain to provide care for those who traveled greater distances for the initial surgical procedure. A standardized protocol was developed for our long-distance patients to minimize variations that exist between the companies and individual orthotic providers. As a result, only orthotists specifically trained in this protocol for postoperative cranial molding were allowed to evaluate and treat our patients. While most orthotists have had experience creating and modifying helmets to treat other cranial abnormalities (e.g., deformational plagiocephaly), these specialists have received additional training in the specifics of helmet therapy following endoscopic release of craniosynostosis. Results are quantitatively assessed,

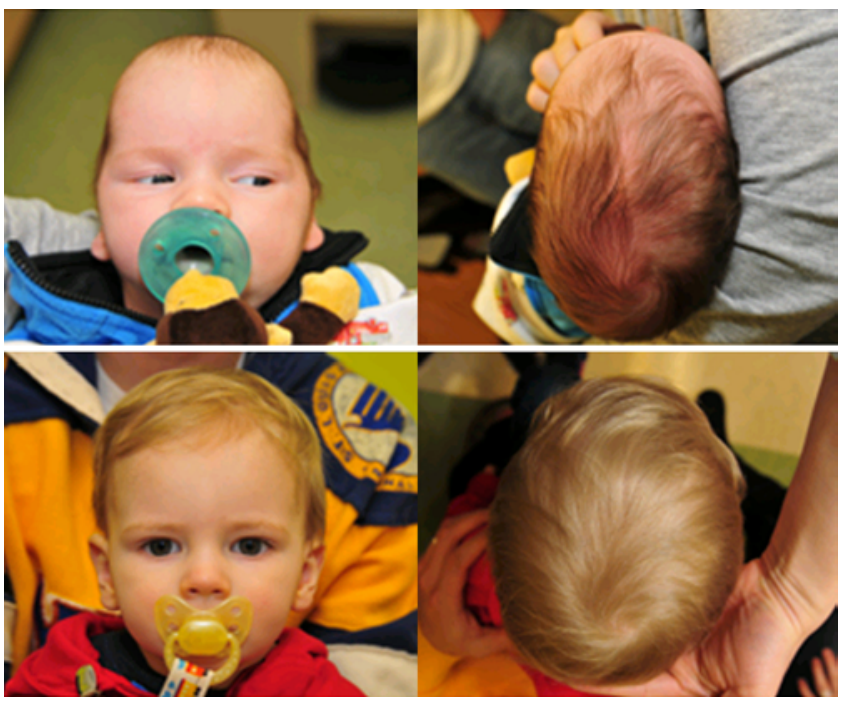

FIG. 3. Upper: Preoperative frontal and vertex views of a 1.6-month-old boy with sagittal craniosynostosis (preoperative $\mathrm{Cl} 66$ ). Lower: The same patient at 1.1 years of age after endoscopic repair and helmet therapy (duration 9.8 months) with a $\mathrm{Cl}$ of 80 . Figure is available in color online only. 
with 3D photographs taken every 3 months to assess improvements in head shape.

\section{Results}

A total of 100 consecutive patients were evaluated for this study, with a male to female ratio of 7:3. No patients within this cohort were excluded from this study, regardless of follow-up or compliance. The patients' age at the time of surgery ranged from 1.6 to 6.9 months, with a mean of $3.3 \pm 1.1$ months (Table 1). All patients underwent endoscopic strip suturectomy; however, 1 patient required conversion to open technique due to the presence of a large emissary vein that was deemed unsafe to manage endoscopically. Two patients were noted to have chromosomal abnormalities (q10 deletion, 19p13.2 deletion). The remaining cases were nonsyndromic.

The perioperative data are summarized in Table 1. The mean $( \pm \mathrm{SD})$ estimated blood loss was $34.0 \pm 34.8 \mathrm{ml}$, and the mean length of hospital stay was $1.1 \pm 0.4$ days (1st tertile 1.3 days, 2 nd tertile 1.1 days, and 3rd tertile 1.0 day). The mean preoperative hemoglobin level ( $9.7 \pm$ $1.1 \mathrm{~g} / \mathrm{dl}$ ) was slightly higher than the mean postoperative discharge hemoglobin level $(8.2 \pm 1.5 \mathrm{~g} / \mathrm{dl})(\mathrm{p}<0.0001)$, and the transfusion rate was $9 \%$. The decision to administer a transfusion to a baby is not taken lightly and is made on a case-by-case basis. Hemodynamic changes associated with a drop in hemoglobin indicated a need for blood product. Patients were safely discharged without complications with a range of hemoglobin levels (5.4-13.1 $\mathrm{g} / \mathrm{dl})$. The mean preoperative and postoperative CI measurements were $69.1 \pm 3.8$ and $77.7 \pm 4.2$, respectively $(\mathrm{p}<$ 0.001 ) (Table 2). The mean value for total operative time was $77.1 \pm 22.2$ minutes, and the operative time gradually became shorter with more experience (and the conversion to narrow-strip craniectomy) (Fig. 4).

Over the course of these 100 patients, there was an upward trend in the postoperative CI; however, the preoperative CI also showed an upward trend (Fig. 5). Analysis of the data reveals that increasing age at time of operation is inversely related to the change in CI measured in our cohort (Fig. 6). The mean duration of helmet therapy was 8.0 \pm 2.9 months, and duration of helmet therapy was not found to correlate with change in CI. Not all patients strictly followed the treatment protocol with their helmet therapy. To provide a fair assessment of the technique, we included all 100 patients regardless of compliance with helmet therapy. Noncompliance was noted in 5 cases (mean preoperative CI $68 \pm 1.3$, mean postoperative CI $77 \pm 4.2$ ). Compliance entailed wearing the helmet for the recommended duration of time and making timely follow-up with the orthotist. In $64 \%$ of cases, the orthosis was provided by the local orthotics provider, Orthotics \& Prosthetics Co., and in the remaining $36 \%$, a nationally available provider, Hanger Clinic, was used. These 2 companies were used to accommodate patients who lived locally as well as those who had traveled from afar for surgery.

The first 63 patients underwent wide-vertex suturectomy, while the following 37 patients underwent the narrowvertex suturectomy; all patients received postoperative helmet therapy. In an age-matched subgroup analysis of 14
TABLE 1. Patient demographic and clinical characteristics

\begin{tabular}{lc}
\hline \multicolumn{1}{c}{ Characteristic } & Value \\
\hline Age (mos) & $3.3 \pm 1.1$ \\
\hline Male:female (\%) & $70: 30$ \\
\hline OR time (mins) & $77.1 \pm 22.2$ \\
\hline Estimated blood loss (ml) & $34.0 \pm 34.8$ \\
\hline Hospital stay (days) & $1.1 \pm 0.4$ \\
\hline No. of patients who received transfusions & 9 \\
\hline
\end{tabular}

$\mathrm{OR}=$ operating room.

Values are presented as mean \pm SD unless otherwise indicated.

wide-vertex and 14 narrow-vertex cases, operative times were $83.4 \pm 18.3$ and $59.0 \pm 16.7$ minutes, respectively $(\mathrm{p}$ $<0.0001$ ). Interestingly, Fig. 4 suggests that there was a gradual reduction in operative time over the course of the 100 cases instead of a dramatic drop between the first 63 cases and the subsequent ones. We attribute this to the learning curve that comes with implementing a slightly different technique-slower at the beginning and gradually improving with experience. There was no statistically significant difference between these 2 groups with respect to percentage change in CI beyond the 6-month postoperative period (Fig. 7). Fourteen patients were selected from each group based on age at operation to allow age matching. In addition, the estimated blood loss did not differ significantly between wide-vertex $(27.5 \pm 13.7 \mathrm{ml})$ and narrow-vertex $(25.4 \pm 6.6 \mathrm{ml})$ cases $(\mathrm{p}=0.60)$.

There was no incidence of major neurological sequelae (i.e., stroke), cardiac events, or death. A total of 2 surgical complications occurred. Conversion from endoscopic to open technique was required in 1 case due to difficulty controlling bleeding from a large emissary vein. This patient required a blood transfusion and had an uneventful postoperative course. The other surgical complication involved a patient who required readmission postoperatively due to emesis and a small subarachnoid hemorrhage discovered on CT scan. This patient did well with observation alone. Three medical complications occurred in the postoperative period: hyperglycemia, respiratory distress requiring CPAP (continuous positive airway pressure), and respiratory stridor from respiratory syncytial virus infection.

\section{Discussion}

The surgical management of infants with sagittal synostosis has traditionally relied on open cranial vault remodeling techniques. However, minimally invasive techniques, including endoscope-assisted craniectomy repair followed by helmet therapy, have been increasingly used

TABLE 2. Comparison of mean pre- and postoperative hemoglobin and $\mathrm{Cl}$ values

\begin{tabular}{lrrl}
\hline \multicolumn{1}{c}{ Variable } & \multicolumn{1}{c}{ Preop } & \multicolumn{1}{c}{ Postop } & p Value \\
\hline Hemoglobin $(\mathrm{g} / \mathrm{dl})$ & $9.7 \pm 1.1$ & $8.2 \pm 1.5$ & $<0.0001$ \\
\hline $\mathrm{Cl}(\%)$ & $69.1 \pm 3.8$ & $78.0 \pm 4.2$ & $<0.001$ \\
\hline
\end{tabular}

Mean values are presented with SDs. 


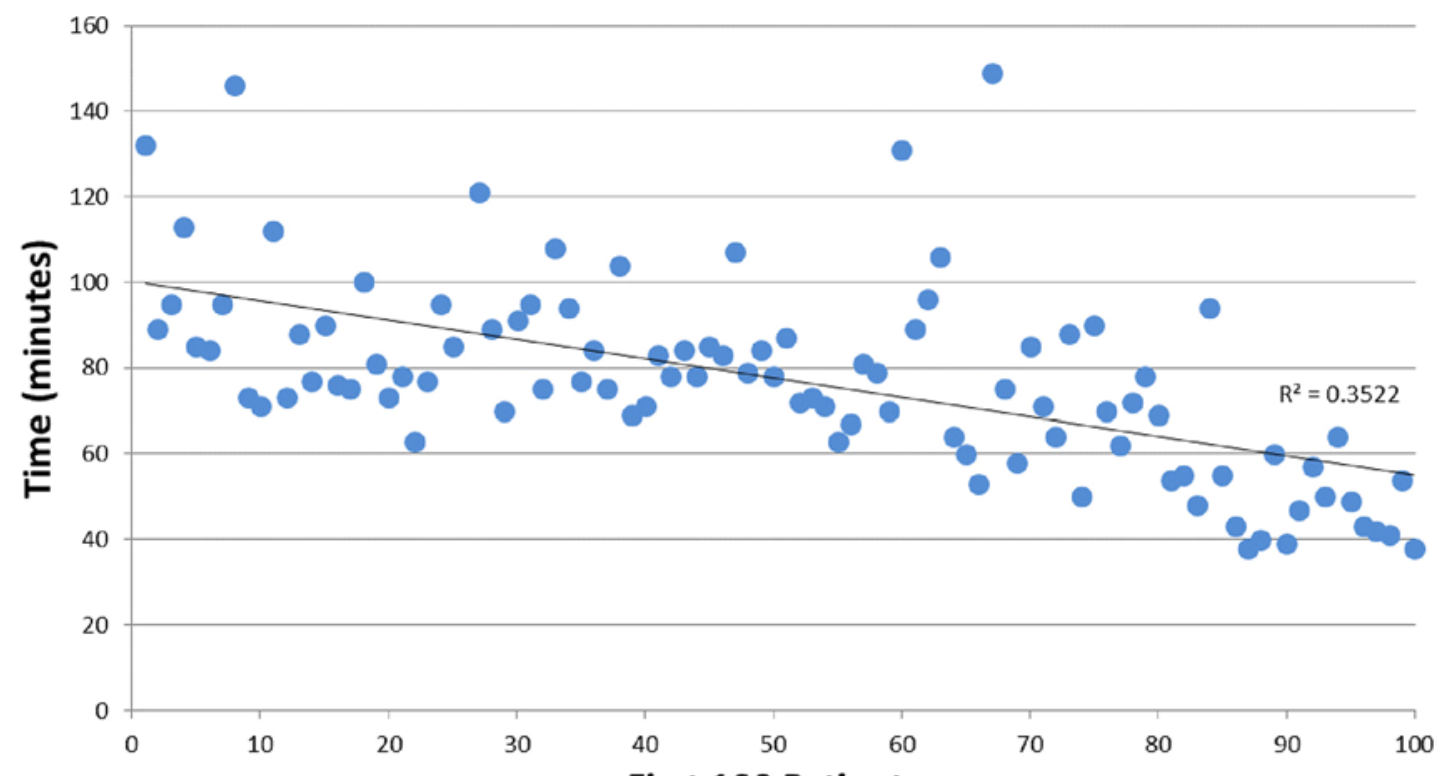

First 100 Patients

FIG. 4. Trend of operative time (in minutes) over the course of 100 consecutive cases. Figure is available in color online only.

to treat the various sutural synostoses. This clinical series of 100 consecutive cases in which patients underwent endoscopic repair of sagittal synostosis shows that it is a safe and effective technique. We also reviewed the evolution in multidisciplinary care of these patients at our single institution.

Clinical outcomes and improvement in CI seen in our population are similar to those seen at other craniofacial centers with endoscopic experience., ${ }^{1,2,10,12,22}$ Of note, 16 patients in this series had a change in CI of 5 or less. In our experience, the degree of change is less important than the final outcome $(\mathrm{CI} \geq 75)$. We offer surgery to patients based on whether they have a prematurely fused sagittal suture or not. We believe that surgery offers the patient the best chances of achieving and maintaining a CI within the normal range. However, the CI is only once facet of the child's overall appearance. There are morphological aberrancies associated with sagittal craniosynostosis that we do not fully understand and may not be normalized with current techniques. The immediate benefits of the endoscopic technique have been well described-operating times and length of hospital stay are shorter, patients require fewer transfusions, and the overall cost is significantly less than with open cranial vault remodeling. ${ }^{24,26}$

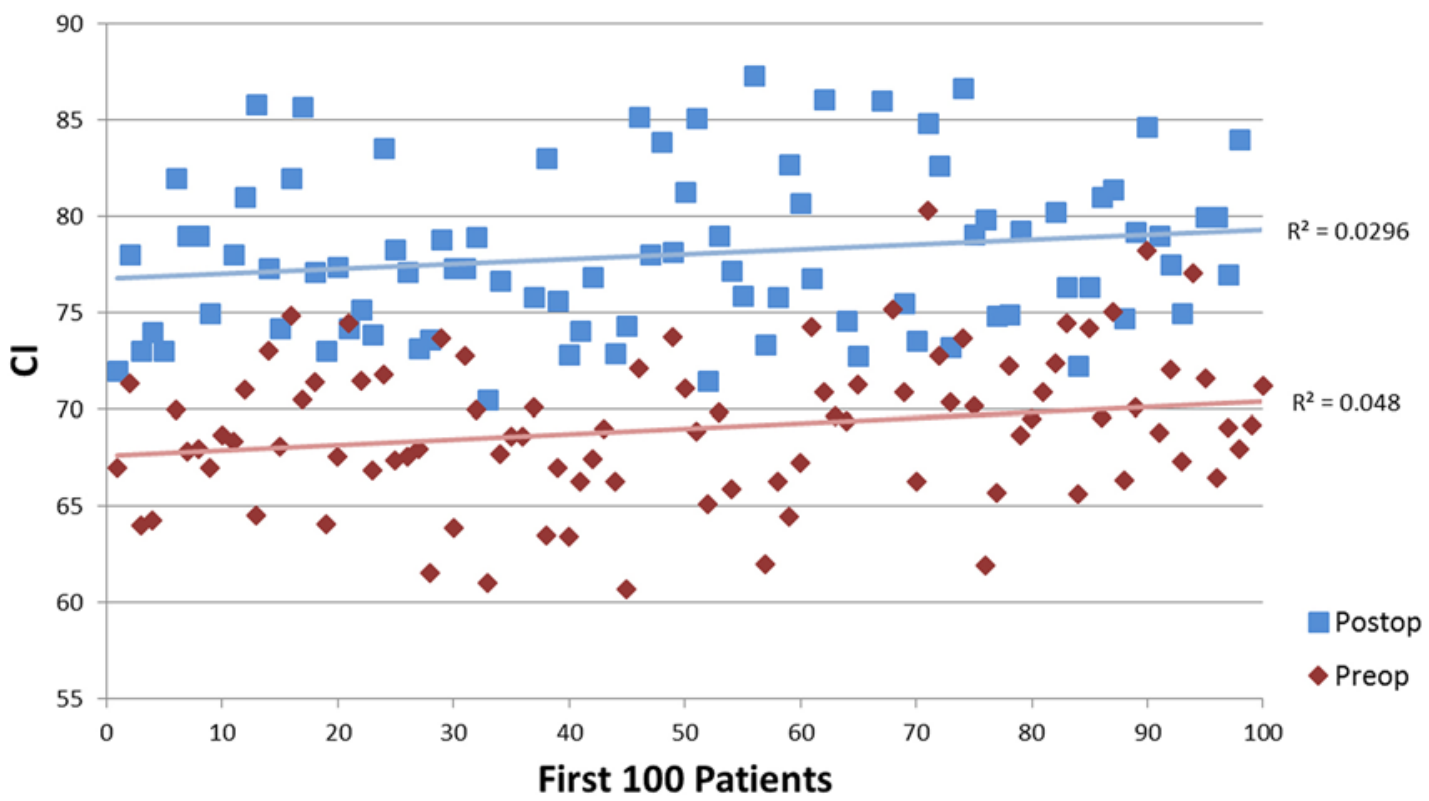

FIG. 5. Preoperative and postoperative Cls. Figure is available in color online only. 


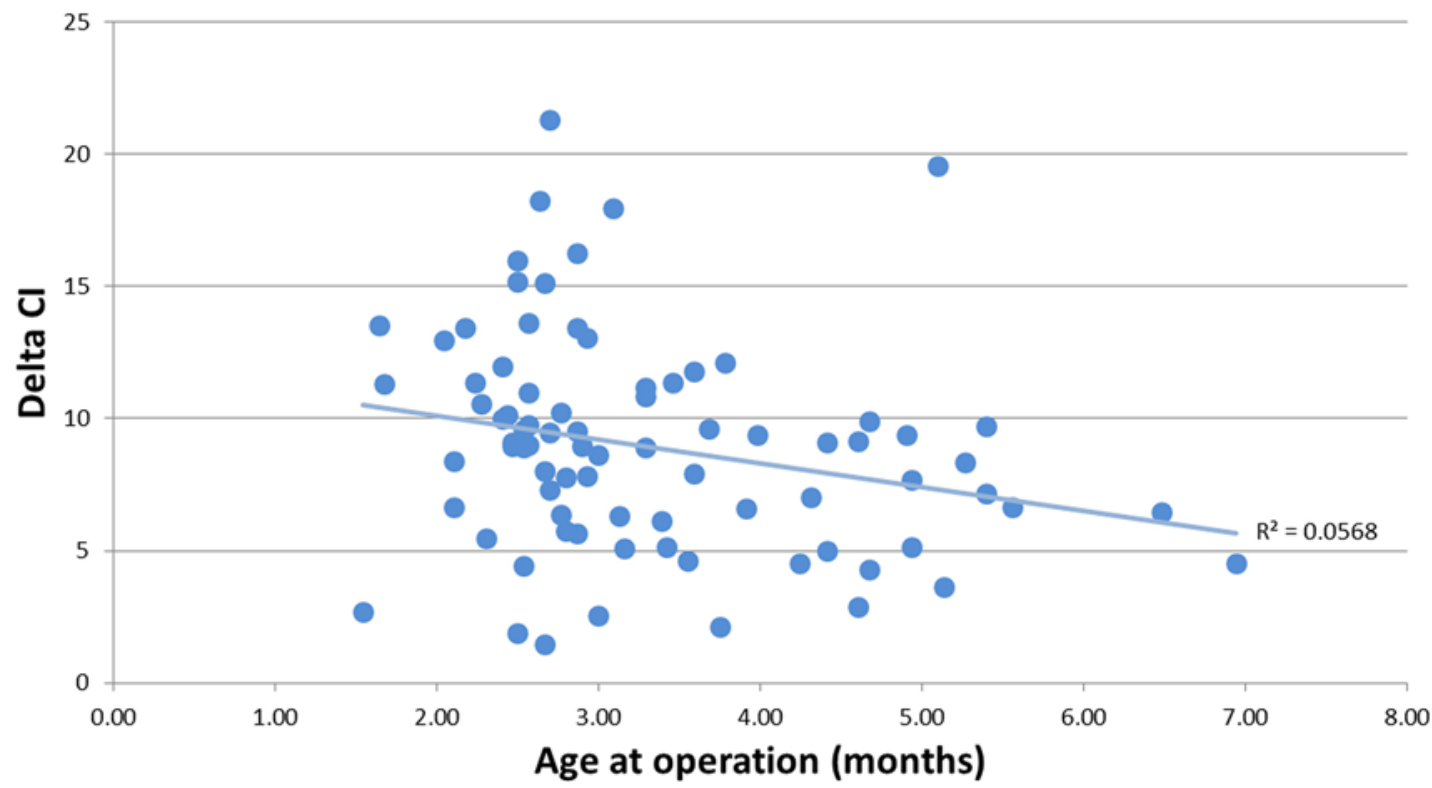

FIG. 6. Operative age (in months) versus change in Cl. Figure is available in color online only.

Over the last decade, our institution has published several studies on this group of patients. In 2011, Shah et al. concluded that endoscope-assisted strip craniectomy offers an effective treatment for sagittal craniosynostosis that is comparable for calvarial vault reconstruction, with no increase in morbidity. ${ }^{24}$ The patients treated with endoscopic technique $(n=47)$ had significantly shorter hospital stay, less blood loss, lower transfusion rates, shorter operating times, and equivalent postoperative CIs (endoscopic 75.9, open 76.9) compared with patients who underwent open surgery $(n=42)$. The patients in the endoscopic group had been followed for 13 months, while those in the open group were followed for 24.6 months.

In a study with longer-term follow-up, Le et al. used 3D photography to show that there was no statistically significant difference in cephalometric measurements (e.g., head circumference, CI, cranial vault volume, cranial height, and forehead inclination) between patients who underwent open repair and endoscopic strip craniectomy with barrelstave osteotomies and helmet therapy at more than 3 years following surgery.$^{18}$ Cost analysis of open and endoscopic cases was performed by Vogel et al. for St. Louis Children's Hospital; ${ }^{26}$ the retrospective case-control study of 21 open cranial vault remodeling cases and 21 cases in which patients were treated with endoscopic craniectomy with helmet therapy showed that the overall costs per case were $\$ 56,990$ and $\$ 37,255$, respectively. This analysis accounted for hospital charges, professional and helmet fees,

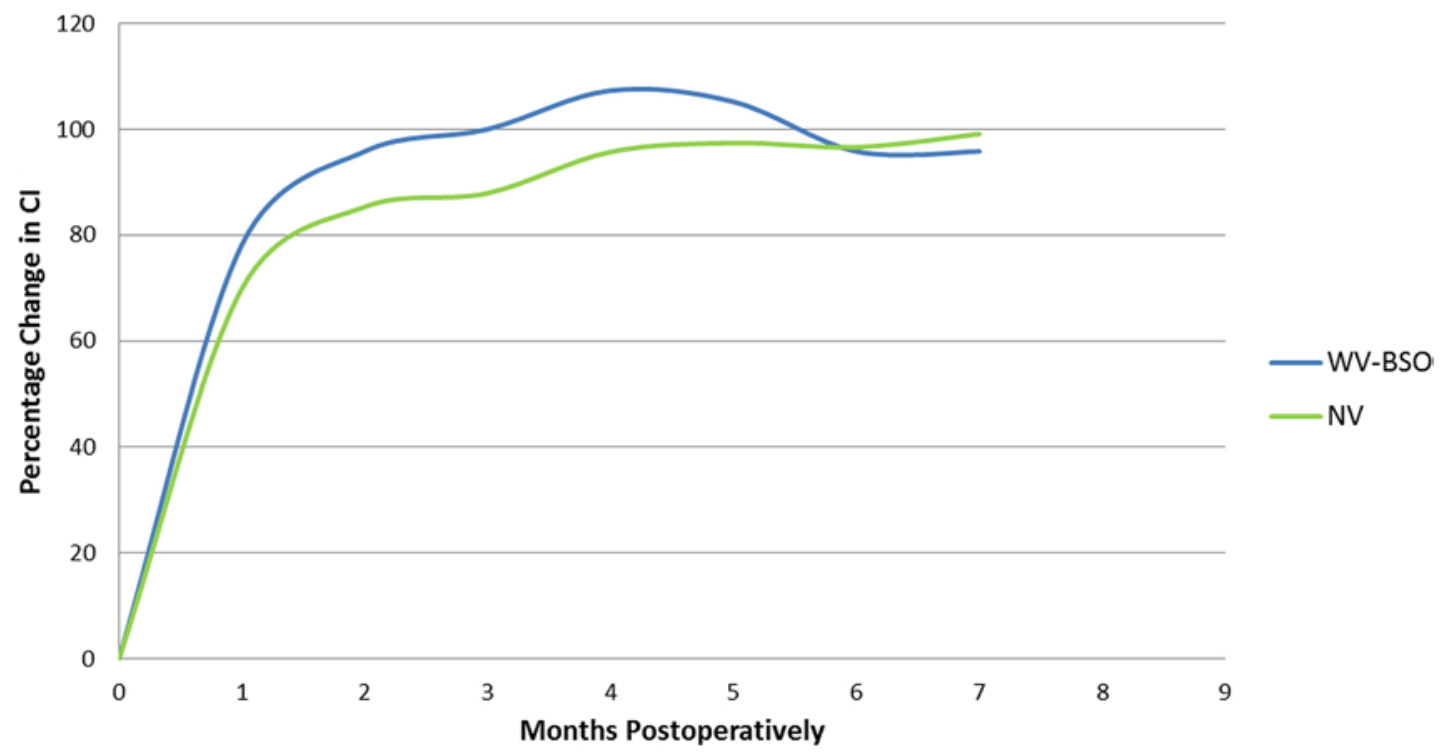

FIG. 7. Percentage change in $\mathrm{Cl}$ between wide-vertex procedures with bilateral-stave osteotomies (WV-BSO) and narrow-vertex (NV) procedures. Figure is available in color online only. 
and clinic visits. The lower utilization of perioperative resources associated with the endoscopic technique suggests that it may be a cost-effective first-line surgical option.

\section{Changes in Surgical Protocols}

Since the inception of endoscope-assisted craniosynostosis repair at our institution, the patient care protocol has undergone several gradual changes. Intraoperatively, we eliminated the placement of arterial lines after the first few cases. This was the result of low blood loss and infrequent transfusion requirements. Less invasive monitoring lines meant less preparation time for the anesthesia providers. It is well documented that the risk of arterial complications (e.g., obstruction, hematoma, infection) is increased with younger patient age and longer duration of cannulation. ${ }^{5}$

The benefits of routine perioperative steroid treatment have been reported for many operations-particularly, open repair of craniosynostosis. In a systematic review by Wei et al., the authors found improved control of postoperative edema, early time to eye opening, and reduced length of hospital stay. ${ }^{27}$ Furthermore, no adverse effects, morbidity, death, or technical errors were associated with steroid administration. However, it was noted that the conclusions were confounded by institutional differences in timing, method, and technique of administration. Han et al. were the first to report on intravenous administration of steroids in a large series of endoscopic repairs. ${ }^{7}$ The authors found that the $6.6 \%$ of patients who received perioperative dexamethasone had a length of stay similar to that of patients who did not receive steroids, with no additional morbidity. In our cohort of sagittal synostosis patients, we favored the use of steroids to allow for the possibility of early helmet application on postoperative Day 1 . While we have not seen problems with the use of intravenously administered dexamethasone, we do not yet have a standardized protocol and our recommendation regarding its use is limited by the varied administration practices of our surgeons.

As with any operation, patient positioning is crucial to optimizing surgical exposure and minimizing iatrogenic injuries to the patient. Early in our practice, following endotracheal induction of general anesthesia, the patients were placed in a prone position on a pediatric beanbag as originally described by Jimenez and Barone. ${ }^{9}$ Subsequently, we transitioned to using a DORO Headrest System (Pro Med Instruments). We found that the head holder provided improved stability while maintaining a secure airway (Fig. 1). Use of this device was favored by the anesthesia providers, due to its ability to optimize stability of the endotracheal tube.

We have been able to remove less cranium using our "narrow-vertex" suturectomy technique without affecting patient safety or outcome with respect to CI. The promising results achieved by colleagues at Boston Children's Hospital with this technique encouraged us to transition away from the "wide-vertex" technique. In this series, the 64th patient was the first to receive the narrow-vertex technique-all subsequent patients underwent the same procedure. One advantage of this technique, as seen in our analysis of age-matched patients, is that it saved approximately 20 minutes of operative and anesthetic time over the wide-vertex technique. Another motivation for using the narrow-vertex technique was to reduce the risk of persistent calvarial defects of larger resections. Though this phenomenon has been described in open repair of craniosynostosis, ${ }^{21}$ no significant literature exists to document the long-term sequelae of cranial defects after endoscopic repair.

The immediate postoperative care of our endoscopic patients was streamlined as well. During the first 3 years of performing this operation, patients were automatically admitted to the pediatric intensive care unit (ICU) for observation-this was in line with our postoperative care of open craniosynostosis repair. It was confirmed that patients who underwent endoscopic repair could be discharged home typically after 1 day of postoperative observation. Our prior data show that the average length of hospital stay was 1.1 days for endoscopically treated patients and 4.67 days for patients who underwent open surgical repair $(p<0.0001){ }^{27}$ Furthermore, since endoscopically treated patients left the operating room extubated and without invasive monitoring (e.g., an arterial line), we admitted these patients directly to a general surgical floor. Patients continued to receive the appropriate level of care, while not burdening the health care resources of the ICU. From an administrative and cost-savings standpoint, the clinical changes resulted in a negligible change in the overall cost of endoscopic technique.

\section{Limitations}

There are a number of limitations to this study. The evolution in the care of our patients with craniosynostosis, in part, involved streamlining the perioperative process. We made changes that we deemed appropriate to improve patient care while reducing unnecessary interventions and costs (e.g., arterial lines, ICU care). Nonetheless, we cannot be certain that these changes did not result in detrimental outcomes that we did not observe and document. Our data show good overall cephalometric and clinical outcomes; however, we did not perform an age-matched control analysis. Age-matched analysis has been previously done, though with smaller numbers, and the results were good..$^{18}$ While the CI is the gold standard for monitoring disease severity and provides us with a convenient means to communicate among clinicians, it remains a crude way to quantify head shape. The intent of this study was to review the result of 100 consecutive endoscopic cases, beginning from program inception. Although slight variations in surgical technique and preference exist between our plastic surgeons, a single pediatric neurosurgeon (M.D.S.) was involved in the operation and care of all of the patients.

Our experience with endoscopic management of sagittal and other forms of craniosynostoses (e.g., metopic, coronal, lambdoid, multisuture) leads us to advocate for postoperative helmeting. However, as seen in this study, a few patients whose caregivers were not compliant with helmeting also achieved CIs over 75. Although not specifically studied here, we previously reported that postoperative head shape cannot fully be appreciated by traditional CI measures alone. ${ }^{6}$ Furthermore, it is possible that we err on the side of overtreatment with helmet therapy, as consensus on the optimal duration of this management protocol has yet to be established. We have not witnessed any 
adverse clinical events with helmet therapy. Our helmeting protocol is, in part, based on our observations during early follow-up (e.g., 1-2 years). Long-term follow-up will be important in helping us establish more definitive treatment plans.

While this study details the perioperative, aesthetic, and promising early clinical outcomes of the endoscopic technique, it does not include neuropsychological data. A small proportion of the patient cohort since 2006 have reached an age appropriate for cognitive testing, but cognitive outcome data are not yet available for the entire cohort. All of the patients are followed closely in a multidisciplinary team setting, which includes evaluation by a developmental psychologist. Long-term neuropsychological results with this population are needed. In this regard, there have been concerns raised about the potential shortcomings of an endoscopic technique. In a multicenter study, Hashim et al. found that patients who underwent whole-vault cranioplasty attained higher intelligence quotient and achievement scores than those treated with strip craniectomy. ${ }^{8}$ It is unclear if the conclusions drawn by these authors are truly applicable to our patients. Our technique is notably different in that our patients did not receive suture constriction in the anterior-posterior dimension and instead received postoperative helmeting. Furthermore, neuropsychological development in children with craniosynostosis is multifactorial (e.g., affected by age, suture type, socioeconomic factors $)^{3}$ and a causal model of surgery type and cognitive outcome with the current data remains tenuous. ${ }^{25}$ Additional studies with appropriate techniques and controls are necessary to provide patients with recommendations and optimal treatment options.

\section{Conclusions}

Early management of sagittal craniosynostosis with an endoscope-assisted technique combined with postoperative helmet therapy is safe and effective. This technique offers the patients a short hospital stay and low perioperative morbidity. We emphasize that compliance with helmet therapy and collaborative care with the orthotists and multidisciplinary team remain the most essential aspects of a successful outcome.

\section{References}

1. Abbott MM, Rogers GF, Proctor MR, Busa K, Meara JG: Cost of treating sagittal synostosis in the first year of life. $\mathbf{J}$ Craniofac Surg 23:88-93, 2012

2. Berry-Candelario J, Ridgway EB, Grondin RT, Rogers GF, Proctor MR: Endoscope-assisted strip craniectomy and postoperative helmet therapy for treatment of craniosynostosis. Neurosurg Focus 31(2):E5, 2011

3. Derderian CA, Heppner C, Cradock MM, Woo AS, Patel KB, Smyth MD, et al: The effects of whole-vault cranioplasty versus strip craniectomy on long-term neuropsychological outcomes in sagittal craniosynostosis. Plast Reconstr Surg 136:114e-115e, 2015

4. Dlouhy BJ, Nguyen DC, Patel KB, Hoben GM, Skolnic GB, Naidoo SD, et al: Endoscope-assisted management of sagittal synostosis: wide vertex suturectomy and barrel stave osteotomies versus narrow vertex suturectomy. J Neurosurg Pediatr 18:674-678, 2016

5. Dumond AA, da Cruz E, Almodovar MC, Friesen RH: Fem- oral artery catheterization in neonates and infants. Pediatr Crit Care Med 13:39-41, 2012

6. Dvoracek LA, Skolnick GB, Nguyen DC, Naidoo SD, Smyth $\mathrm{MD}$, Woo AS, et al: Comparison of traditional versus normative cephalic index in patients with sagittal synostosis: measure of scaphocephaly and postoperative outcome. Plast Reconstr Surg 136:541-548, 2015

7. Han RH, Nguyen DC, Bruck BS, Skolnick GB, Yarbrough CK, Naidoo SD, et al: Characterization of complications associated with open and endoscopic craniosynostosis surgery at a single institution. J Neurosurg Pediatr 17:361-370, 2016

8. Hashim PW, Patel A, Yang JF, Travieso R, Terner J, Losee JE, et al: The effects of whole-vault cranioplasty versus strip craniectomy on long-term neuropsychological outcomes in sagittal craniosynostosis. Plast Reconstr Surg 134:491-501, 2014

9. Jimenez DF, Barone CM: Endoscopic craniectomy for early surgical correction of sagittal craniosynostosis. J Neurosurg 88:77-81, 1998

10. Jimenez DF, Barone CM: Endoscopic technique for sagittal synostosis. Childs Nerv Syst 28:1333-1339, 2012

11. Jimenez DF, Barone CM, Cartwright CC, Baker L: Early management of craniosynostosis using endoscopic-assisted strip craniectomies and cranial orthotic molding therapy. Pediatrics 110:97-104, 2002

12. Jimenez DF, Barone CM, McGee ME, Cartwright CC, Baker CL: Endoscopy-assisted wide-vertex craniectomy, barrel stave osteotomies, and postoperative helmet molding therapy in the management of sagittal suture craniosynostosis. J Neurosurg 100 (5 Suppl Pediatrics):407-417, 2004

13. Justice CM, Yagnik G, Kim Y, Peter I, Jabs EW, Erazo M, et al: A genome-wide association study identifies susceptibility loci for nonsyndromic sagittal craniosynostosis near BMP2 and within BBS9. Nat Genet 44:1360-1364, 2012

14. Kanev PM, Lo AK: Surgical correction of sagittal craniosynostosis: complications of the pi procedure. J Craniofac Surg 6:98-102, 1995

15. Khechoyan D, Schook C, Birgfeld CB, Khosla RK, Saltzman $\mathrm{B}$, Teng CC, et al: Changes in frontal morphology after single-stage open posterior-middle vault expansion for sagittal craniosynostosis. Plast Reconstr Surg 129:504-516, 2012

16. Lane LC: Pioneer craniectomy for relief of mental imbecility due to premature sutural closure and microcephalus. JAMA 18:49-50, 1892

17. Lannelongue M: De la craniectomie dans la microcephalie. Compt Rend Seances Acad Sci 110:1382-1385, 1890

18. Le MB, Patel K, Skolnick G, Naidoo S, Smyth M, Kane A, et al: Assessing long-term outcomes of open and endoscopic sagittal synostosis reconstruction using three-dimensional photography. J Craniofac Surg 25:573-576, 2014

19. Mehta VA, Bettegowda C, Jallo GI, Ahn ES: The evolution of surgical management for craniosynostosis. Neurosurg Focus 29(6):E5, 2010

20. Nguyen DC, Patel KB, Skolnick GB, Naidoo SD, Huang AH, Smyth MD, et al: Are endoscopic and open treatments of metopic synostosis equivalent in treating trigonocephaly and hypotelorism? J Craniofac Surg 26:129-134, 2015

21. Prevot M, Renier D, Marchac D: Lack of ossification after cranioplasty for craniosynostosis: a review of relevant factors in 592 consecutive patients. J Craniofac Surg 4:247-256, 1993

22. Ridgway EB, Berry-Candelario J, Grondin RT, Rogers GF, Proctor MR: The management of sagittal synostosis using endoscopic suturectomy and postoperative helmet therapy. J Neurosurg Pediatr 7:620-626, 2011

23. Sgouros S, Hockley AD, Goldin JH, Wake MJ, Natarajan K: Intracranial volume change in craniosynostosis. J Neurosurg 91:617-625, 1999 
24. Shah MN, Kane AA, Petersen JD, Woo AS, Naidoo SD, Smyth MD: Endoscopically assisted versus open repair of sagittal craniosynostosis: the St. Louis Children's Hospital experience. J Neurosurg Pediatr 8:165-170, 2011

25. Speltz ML, Birgfeld C, Starr JR, Collett B, Kapp-Simon K: The effects of whole-vault cranioplasty versus strip craniectomy on long-term neuropsychological outcomes in sagittal craniosynostosis. Plast Reconstr Surg 135:646e-647e, 2015

26. Vogel TW, Woo AS, Kane AA, Patel KB, Naidoo SD, Smyth MD: A comparison of costs associated with endoscope-assisted craniectomy versus open cranial vault repair for infants with sagittal synostosis. J Neurosurg Pediatr 13:324-331, 2014

27. Wei AT, Madsen C, Al-Sheemy A, Kumar AR: Does perioperative steroid use improve clinical outcomes in open repair of craniosynostosis? J Craniofac Surg 26:226-231, 2015

\section{Disclosures}

Dr. Naidoo and Dr. Patel report serving as speakers for Orthomerica and Hanger Clinic. Dr. Patel also reports a consultant relationship with Stryker.

\section{Author Contributions}

Conception and design: Nguyen, Smyth, Kane, Woo. Acquisition of data: Nguyen, Naidoo, Smyth, Kane, Woo. Analysis and interpretation of data: all authors. Drafting the article: all authors. Critically revising the article: all authors. Reviewed submitted version of manuscript: all authors. Approved the final version of the manuscript on behalf of all authors: Nguyen. Statistical analysis: all authors. Administrative/technical/material support: Nguyen, Farber, Skolnick, Smyth, Kane, Woo. Study supervision: Nguyen, Farber, Skolnick, Smyth, Kane, Woo.

\section{Supplemental Information} Previous Presentations

Portions of this work were previously presented at the 94th Annual Meeting of the American Association of Plastic Surgeons in Scottsdale, Arizona, April 11-14, 2015.

\section{Correspondence}

Dennis C. Nguyen, Division of Plastic and Reconstructive Surgery, Washington University School of Medicine, 660 South Euclid Ave., Northwest Tower, Ste. 1150, St. Louis, MO 63110. email: nguyend@wudosis.wustl.edu. 